\title{
Evaluation of the Acceptance of the Hot Mix Asphalt Paving Mixture Using Backpropagation Artificial Neural Network
}

\author{
Mohammed Y. Taha \\ University of Mosul- Engineering College
}

\begin{abstract}
The asphalt content in hot mix asphalt paving mixture is a key factor in producing quality pavements. In recent years, the artificial neural networks approach has attracted wide attention and found a growing number of pavement applications. This paper explores the feasibility of using the backpropagation artificial neural network with sigmoid function as activation function by MATLAB 7.8 software to determine the acceptability of the hot mix asphalt paving mixtures based on the percent of asphalt content and aggregate gradation using their Marshall properties. Several networks architectures, using two hidden layers with different numbers of nodes, are tested to obtained the best results.

The results showed that the network (10-20-10-3) had the best performance, and this network can be used as appropriated method for determining the asphalt content and aggregate gradation acceptability of hot mix asphalt paving mixture. This work concludes that the artificial neural network is a good method which can reduce the time consumed and can be used as a tool in evaluating the hot mix asphalt paving mixtures.
\end{abstract}

Key Words: backpropagation artificial neural network, hot mix asphalt, asphalt content, aggregate gradation, Marshall properties.
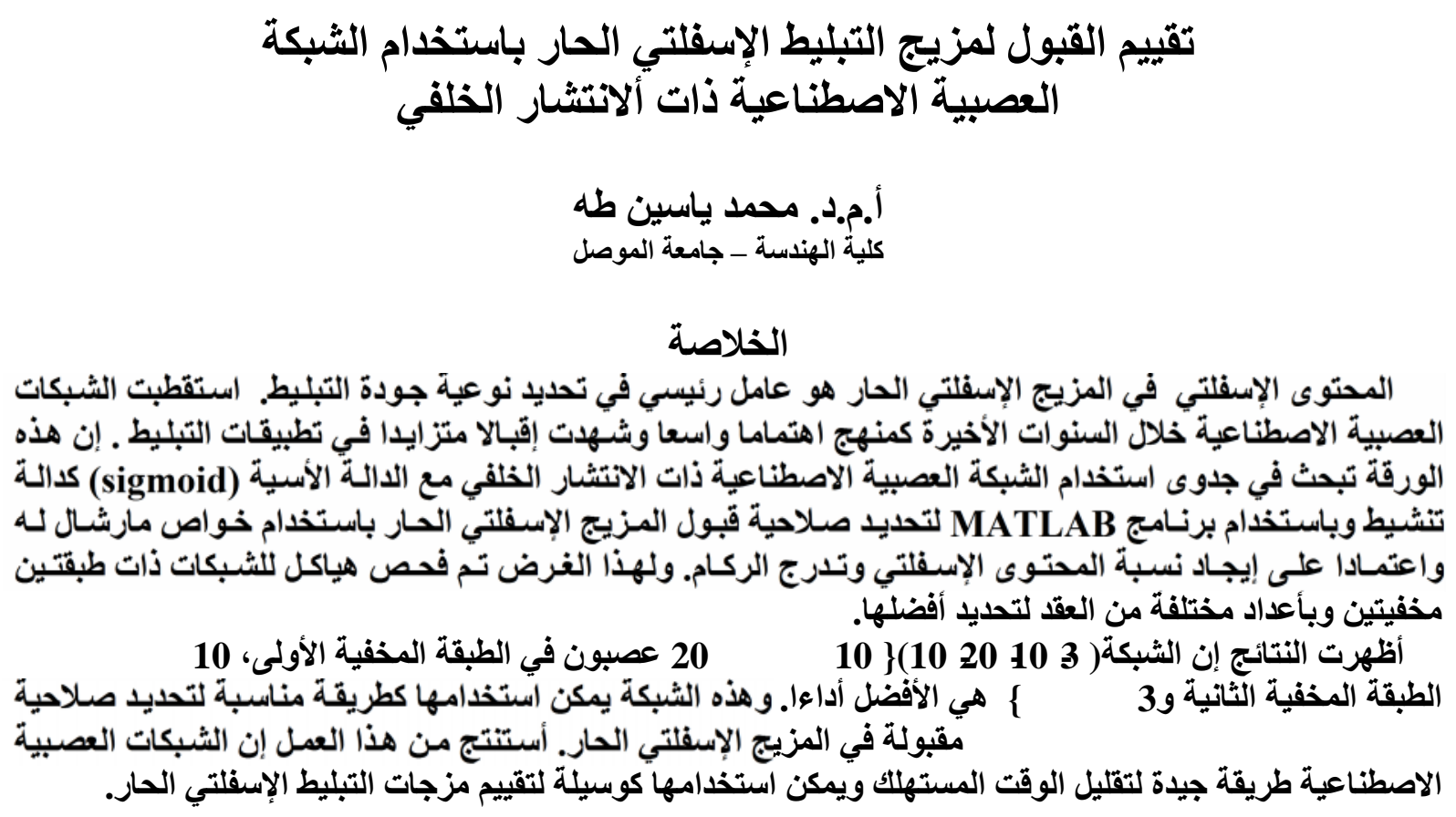

Received : 23-3-2010

Excepted : $18-5-2010$ 


\section{Introduction}

Today, there is an increasing growth of road transportation in the world. Roadways are very large, in volume, in extent, and in value [1]. More than $95 \%$ of paved roads in Iraq are paved with hot mix asphalt (HMA) paving mixtures. HMA is a mixture of asphalt cement and aggregate. The aggregate sum 93-97 percent by weight of the total mixture, while the remaining 3-7 percent is asphalt binder. HMA is a rather complex material upon which many different, and sometimes conflicting, performance demands are placed. In order to meet these demands, three variables must be considered: aggregate, asphalt binder, and the ratio of the asphalt binder to aggregate (asphalt content) [2].

Kind of HMA mix design is the process of determining what asphalt binder to use, what aggregate to use and what the optimum combination of these two ingredients ought to be. Mix design is a laboratory simulation. It is meant to simulate actual HMA manufacturing, construction and performance to the extent possible[2]. The Marshall method of HMA design is the most widely used and was the design method recognized by the Iraqi authorities.

Artificial Neural Networks (ANN) has a wide variety of applications in many disciplines of engineering as well as non engineering analysis [3]. ANNs have been successfully used for tasks involving pattern recognition, function approximation, optimization, forecasting, data retrieval, and automatic control, to name just a few [4, 5]. In recent years many researches have applied the use of ANN in an effort to improve evaluation and prediction of corrective measures [3]. The backpropagation network is a very popular model in ANNs. Backpropagation ANNs are very powerful and versatile networks that can be taught a mapping from one data space to another using a representative set of patterns to be learned $[5,6,7]$.

\section{Objective of Research}

Today, for determining asphalt cement content and aggregate gradation acceptability of HMA mixtures, many Iraqi laboratories use solvents to separate the asphalt cement from the aggregate in HMA mixtures. These solvents used for extraction tests are expensive, difficult to dispose, and unsafe.

This work primarily focuses on the development of ANN models using MATLAB 7.8 software, as quality control toll for HMA mixtures, based on feed-forward backpropagation network with a sigmoid function as activation function to predict the asphalt content and aggregate gradation acceptability of HMA mixtures as output data using Marshall properties and specification requirements of these mixtures as input data.

\section{Artificial Neural Networks Applications}

ANN approach is a valuable computational tool that is increasingly being used to solve resource- intensive complex problems as alternative to using traditional numerical and statistical techniques $[3,4,8]$. This is because ANN is inherently better in analyzing non-linear behavior. The advantage of neural ANN is derived from their capability to learn from examples they are trained with and have the further capability of generalizing beyond the examples learned [3,9].

There are many studies of using ANN in pavement distress $[3,4,10,11,12]$, pavement structural modeling $[1,13,14,15]$, pavement performance, analysis and design $[16,17,18,19,20]$, pavement rehabilitation and maintenance $[6,21,22]$, pavement materials and properties $[23,24,25,26]$, and pavement management $[27,28,29,30]$. 


\section{Backpropagation}

The backpropagation network is probably the most well known and widely used among the current types of ANN systems available. It is a multilayer feed-forward network with a different transfer function in the artificial neuron and more powerful learning rule [8]. The backpropagation ANNs have been described in many sources $[1,3,7,8,31,32]$. Backpropagation ANNs are fully connected, layered, feed-forward networks in which activation flow from the input layer through the hidden layer and then to the output layer. The network usually starts with a set of random weights and adjusts its weights according to each learning example. Each learning example is passed through the network to activate the nodes. The actual output of the network is then compared with the target output, and the error estimates are then propagated back to the hidden and input layers. There have been several successful studies of using backpropagation ANNs in pavement analysis, performance, and design concepts [ $1,3,6,11,12,15,26,27,28]$.

\section{Backpropagation Algorithm}

The backpropagation algorithm is essentially a technique that minimizes the network error function as formulated below $[3,7,8,31,32]$ :

1. Weight Initialization:

Set all weights and node threshold to small random numbers. Note that the node threshold is the negative of the weight from the bias unit.

2. Calculation of Activation:

a) The activation level $f$ an input unit is determined by the instance presented to the network.

b) The activation level $O_{\mathrm{j}}$ of a hidden and output unit is determined by:

$O_{\mathrm{j}}=F\left(\sum W_{\mathrm{ji}} O_{\mathrm{i}}-\theta_{\mathrm{j}}\right)_{\mathrm{i}}$

Where $\mathrm{i}$ vary from 0 to $\mathrm{N}$ input neurons, $\mathrm{j}$ varies from 1 to $\mathrm{L}$ hidden neurons, $W_{\mathrm{ji}}$ is the weight from an input $O_{\mathrm{i}}, \theta_{\mathrm{j}}$ is the node threshold, and $F$ is the sigmoid function:

$F(\mathbf{a})=1 /\left(1+\mathrm{e}^{-\mathrm{a}}\right)$

3. Weight Training:

a) Start at the output units and work backward to the hidden layers recursively. Adjust weights by:

$W_{\mathrm{ji}}(\mathbf{t}+\mathbf{1})=W_{\mathrm{ji}}(\mathrm{t})+\Delta W_{\mathrm{ji}}$

Where $W_{\mathrm{ji}}(\mathrm{t})$ is the weight from unit $\mathrm{i}$ to unit $\mathrm{j}$ at time $\mathrm{t}$ and $\Delta W_{\mathrm{ji}}$ is the weight adjustment.

b) The weight change is computed by:

$\Delta W_{\mathrm{ji}}=\eta \delta_{\mathrm{j}} O_{\mathrm{i}}$

Where $\eta$ is a trial independent learning rate $(0<\eta<1)$ and $\delta_{j}$ is the error gradient at unit $\mathbf{j}$. Convergence is sometimes faster by adding a momentum $\operatorname{term}(\alpha)$ : 
$W_{\mathrm{ji}}(\mathrm{t}+1)=W_{\mathrm{ji}}(\mathrm{t})+\eta \delta_{\mathrm{j}} O_{\mathrm{i}}+\alpha\left\{W_{\mathrm{ji}}(\mathrm{t})-W_{\mathrm{ji}}(\mathrm{t}-1)\right\}$

Where $0<\alpha<1$.

c) The error gradient is given by:

* For the output units:

$\delta_{\mathrm{j}}=O_{\mathrm{j}}\left(1-O_{\mathrm{j}}\right)\left(T_{\mathrm{j}}-O_{\mathrm{j}}\right)$

Where $T_{\mathrm{j}}$ is the desired (target) output activation and $O_{\mathrm{j}}$ is the actual output activation at output unit $j$.

* For the hidden units:

$\delta_{\mathrm{j}}=O_{\mathrm{j}}\left(1-O_{\mathrm{j}}\right) \sum \delta_{\mathrm{k}} W_{\mathrm{kj}}$

Where $\delta_{k}$ is the error gradient at unit $k$ to which a connection points from hidden unit $\mathbf{j}$, $k$ varies from 1 to $M$ output neurons.

d) Repeat iterations until convergence in terms of the selected error criterion. An iteration includes presenting an instance, calculating activations, and modifying weights.

As the iteration progress, the network repeatedly cycles through the training set. The parameters $\eta$ and $\alpha$ help provide an accurate approximation of the unknown mean squared error (MSE) minimum. Iterations must be continued until an apparent decrease in the maximum MSE to an acceptable level is observed:

$\mathrm{MSE}=(1 / \mathrm{M.P}) \sum \sum\left(\underset{\mathbf{1}}{T_{\mathrm{kj}}}-O_{\mathrm{kj}}\right)^{2}$

Where $P$ is the total number of training patterns and $M$ is total number of epochs. The following steps show how to derive the equation (4):

- The backpropagation procedure minimizes the error criterion $E$ :

$E=0.5 \sum\left(T_{\mathrm{j}}-O_{\mathrm{j}}\right)^{2}$

But:

$\Delta W_{\mathrm{ji}}=-\eta\left(\partial E / \partial W_{\mathrm{ji}}\right)$

- By using the chain rule, we obtain:

$\partial E / \partial W_{\mathrm{ji}}=\left(\partial E / \partial O_{\mathrm{j}}\right)\left(\partial O_{\mathrm{j}} / \partial W_{\mathrm{ji}}\right)$

- In the case when unit $\mathbf{j}$ is an output unit:

$\delta_{\mathrm{j}}=\left(T_{\mathrm{j}}-O_{\mathrm{j}}\right) \mathrm{F}_{\mathrm{j}}{ }^{\left(\text {net }_{\mathrm{j}}\right)}$

$\partial E / \partial O_{\mathrm{j}}=-\left(T_{\mathrm{j}}-O_{\mathrm{j}}\right)$ 


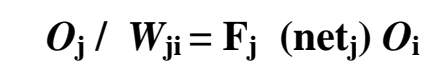

Where: net $_{\mathrm{j}}=\sum_{\mathrm{i}} W_{\mathrm{ji}} O_{\mathrm{i}}$

- Thus, $\quad \partial E / \partial W_{\mathrm{ji}}=-\delta_{\mathrm{j}} O_{\mathrm{i}}$

So, we obtain: $\Delta W_{\mathrm{ji}}=\eta \delta_{\mathrm{j}} O_{\mathrm{i}}$

\section{MATLAB Software}

The MATLAB 7.8 software was used for developing the backpropagation ANNs. MATLAB is a high-performance language for technical computing, it integrates computation, visualization, and programming in an easy-to-use environment, where problems and solutions are expressed in familiar mathematical rotation. MATLAB is an interactive system whose basic data element is an array that dose not required dimensioning (an array-oriented language). This allows formulating solution to many problems $[8,33]$.

\section{Network Architecture:}

One of the most important issues in the development of an ANN model is its architecture, i.e. the determination of the input and output variables, number of hidden layers, and number of neurons (nodes) in each hidden layer. The architecture of the ANN model has significant effect on the success of the developed model [5,9,31].

Usually, an ANN with too few hidden layers is unable to learn sufficiently from training data set, whereas an ANN with too many hidden layers will allow the network to memorize the training set instead of generalizing the required knowledge for unseen patterns[ 29 ]. Haykin [ 5 ] recommends using two hidden layers, the first one for extracting local features and second one for extracting global features.

The number of neurons (nodes) in the middle (hidden) layers can be varied depending on the complexity of the problem and size of the input information. Nelson and Illingworth recommended that a maximum of 4 nodes in the hidden layer be used for each input node $[3,4]$. The results of many studies $[6,29]$ are apparent that there is no significant advantage of using more than three hidden layers. Therefore, the number of hidden layers are varied from 1 to 3 with each layer having between 1 and 4 nodes for each input node.

A network with two hidden layers( 4-layered network) was used in this study. The typical backpropagation network used in this study is shown in Figure 1. This network has one input layer, one output layer, and two hidden layers between them. So several two hidden layer architectures are studied and tested to obtain the best results with the node threshold (bias) $\theta_{\mathrm{j}}$ equal to one. The performance criterion used in this study focuses on the measured in number of iterations and mean squared error (MSE). For each run, a failure occurs when the network exceed the maximum iteration limit of 10000 epochs. Six ANNs were proposed. These networks had two hidden layers of different number of nodes and sigmoid activation function was used for all nodes. While, other network parameters were kept same. Figure 2 depicts the MSE for the proposed networks with respect to the output parameters. Where the number of epochs run of 2000 and learning rate of 0.3 were fixed. 
Finally, the best results are obtained by an 4-layered of 10-20-10-3 network structure, i.e. 10 nodes in the input layer, 20 nodes in the first hidden layer, 10 nodes in the second hidden layer, and 3 nodes in the output layer.

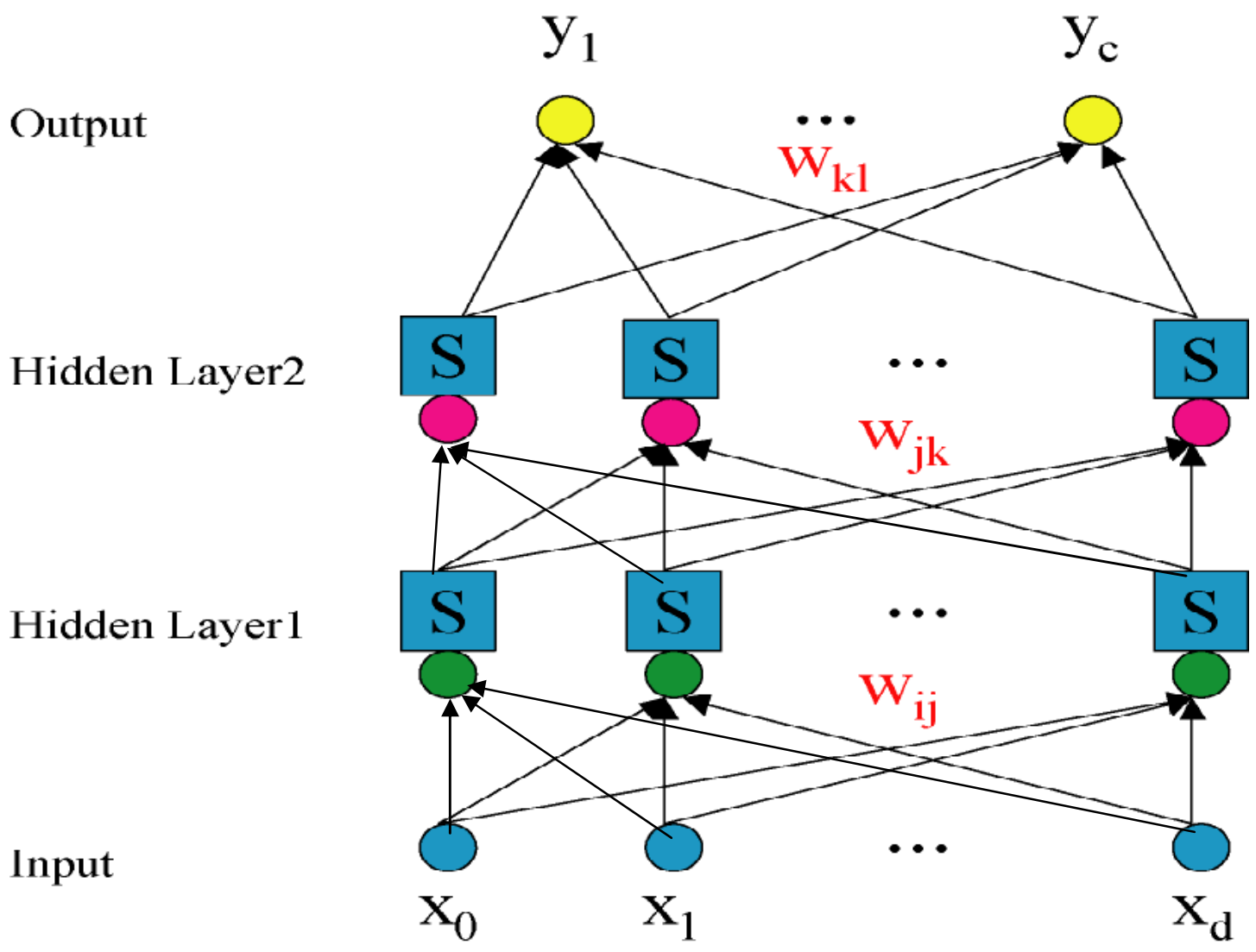

Figure 1. Typical Backpropagation Artificial Neural Netwok.

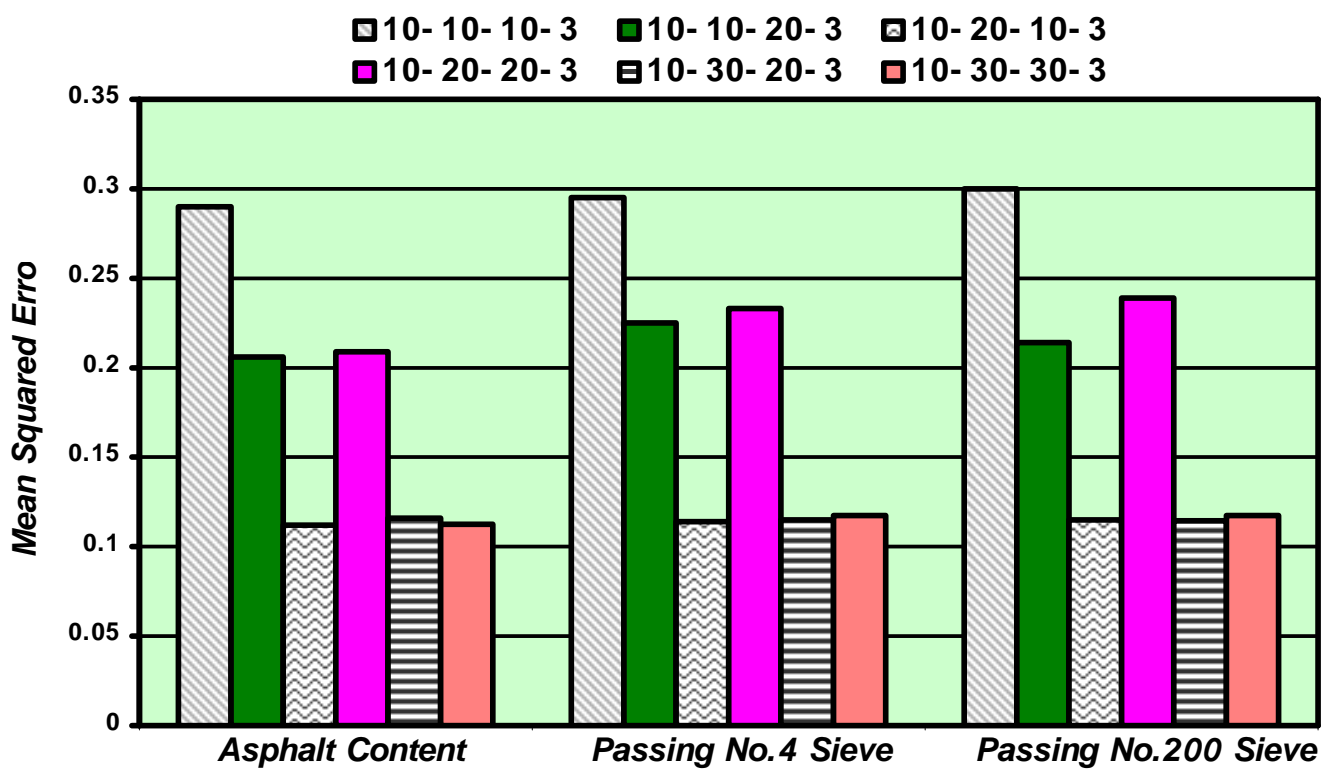

Figure 2. Mean Squared Error for Different Two Hidden Layers Networks According to Output Parameters. 


\section{Study Data}

Certain conditions must be satisfied to meet the success of the study model [1,3]: there should be a large number of input variables and large amount of data that can be used for both training and testing, and the effect of the input variables as well as the interaction among them should not be straight-forward.

The available of 350 data records ( use both existing HMA data available and gathered from laboratory tests curried out from 2005 to 2010 in Highway Lab.) are used by MATLAB 7.8 software to develop the ANN . Where the data are normalized to fall between 0 and 1. Normalization is a common technique used in backpropagation network as the sigmoid transfer function work best for values that lie between 0 and 1 . These data are divided into two sets:

1. Training set: used to determine the network weights, where 225 records are used to develop the network.

2. Testing set: used to verify the effectiveness of the stop criterion and to overcome network over-fitting, then to estimate the network performance. Remaining 125 records are used as input for testing the trained ANN, also these records helped for validating the network.

In this work, the input layer will have 10 nodes corresponding to the Marshall Properties and requirements of HMA mixtures. All these properties must meet the requirements of the General Specification for Roads and Bridges in Iraq for binder and surface courses [34]:

1. Acceptable Marshall Stability.

2. Acceptable Marshall Flow.

3. Acceptable percent of air voids.

4. Maximum aggregate size.

5. Upper limits of percent asphalt content.

6. Lower limits of percent asphalt content.

7. Upper limits of percent passing No.4 sieve.

8. Lower limits of percent passing No.4 sieve.

9. Upper limits of percent passing No.200 sieve.

10. Lower limits of percent passing No.200 sieve.

Marshall stability test is used in accordance with ASTM D1559[35], which measures the first two parameters. For the third parameter, (\% air voids), two different measures of densities of HMA mixture are taken in accordance with ASTM D2726 [35]: Bulk specific gravity and theoretical maximum specific gravity. The last seven input parameters are based on Iraqi standard specifications or job mix formula requirements. Critical sieves, as input parameters, for quality control of HMA mixtures tend to be the maximum aggregate size, the $4.75 \mathrm{~mm}$ (No.4), and $0.075 \mathrm{~mm}$ (No.200) sieve.

The output layer will have 3 nodes corresponding to certain quality characteristics of HMA mixtures, as quality control parameters:

1. Predictive percent asphalt content.

2. Predictive percent passing No.4 sieve.

3. Predictive percent passing No.200 sieve.

In this study, to meet the acceptability of the HMA mixtures based on aggregate gradation (output parameters), the values of percent passing the $4.75 \mathrm{~mm}$ (No.4)sieve and the $0.075 \mathrm{~mm}(\mathrm{No.200})$ sieve are used, The analysis of these two critical sieves will provide a good indication of the effect on the remaining sizes. The target error for each output parameter shall be less than the allowed tolerance in accordance with Iraqi general specifications[34]. 


\section{Results and Discussion}

As shown in Figure 3, the output parameter results of the selected network (10-2010-3) run are present as a square matrix of dimension $3 \times 3$. The values of the nine elements of this matrix are designed to be 0 or 1 . Where the diagonal elements, $a_{11}, a_{22}$ and $\mathbf{a}_{33}$, are represent the output parameters, predictive percent asphalt content, predictive percent passing No.4 sieve and predictive percent passing No.200 sieve respectively. Also, each value of these three elements refers to the acceptability of that parameter in the tested HMA mixture, i. e. passed para- meter when diagonal element value equal to 1 and failed when value equal to 0 .
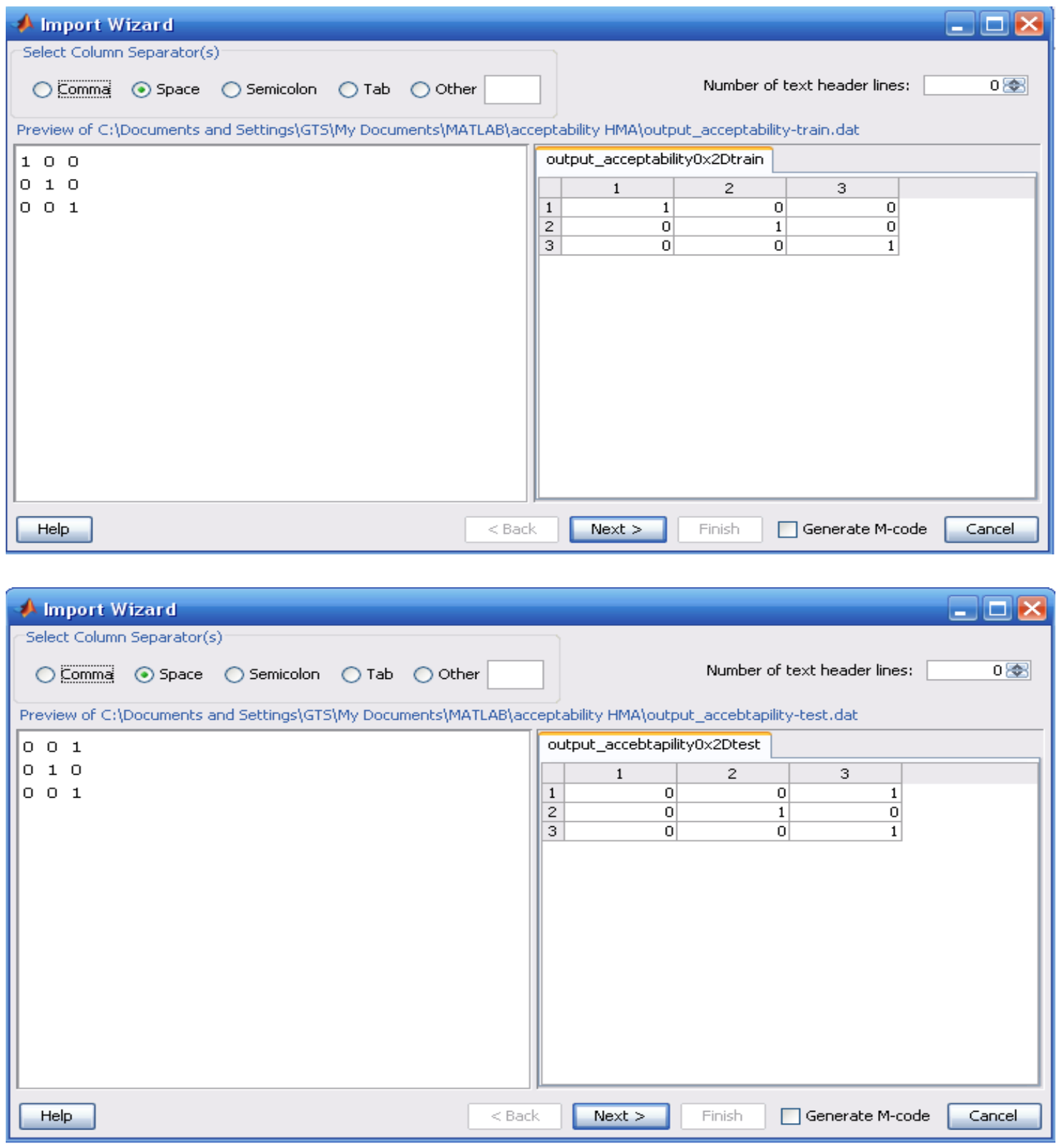

Figure 3. Samples of the Study Network Output Results

The acceptability of any HMA mixture is determined according to the values of the matrix elements. If only the diagonal elements are each equalto unity while all offdiagonal elements are zero (identify matrix), the mixture is meet the required 
specifications and the mixture is passed. Otherwise the mixture is failed. The cause of each failure can be recorded according to:

1. The diagonal element that having the value equal to zero refers to the deficiency of the specific output parameter to meet the requirements.

2.The off-diagonal element that having value equal to unity refers to the type of deficiency. When this off-diagonal element is below the diagonal, the result of the specific output parameter is less than the lower limit of the requirement. But, when this off-diagonal element is up the diagonal, the result of the specific output parameter is more than the upper limit of the requirement.

Figure 4 shows the MSE values for all output parameters increased slightly as the learning rate increased from 0.10 to 0.25 . No noticeable change in the magnitude of the MSE values for learning rates between 0.25 to 0.4 . When learning rate increased above 0.40, the MSEs decreased slightly. Also, this figure indicates that the MSE values for all output parameters remained between 0.10 and 0.12 . Therefore, learning rate of 0.30 was used in the study.

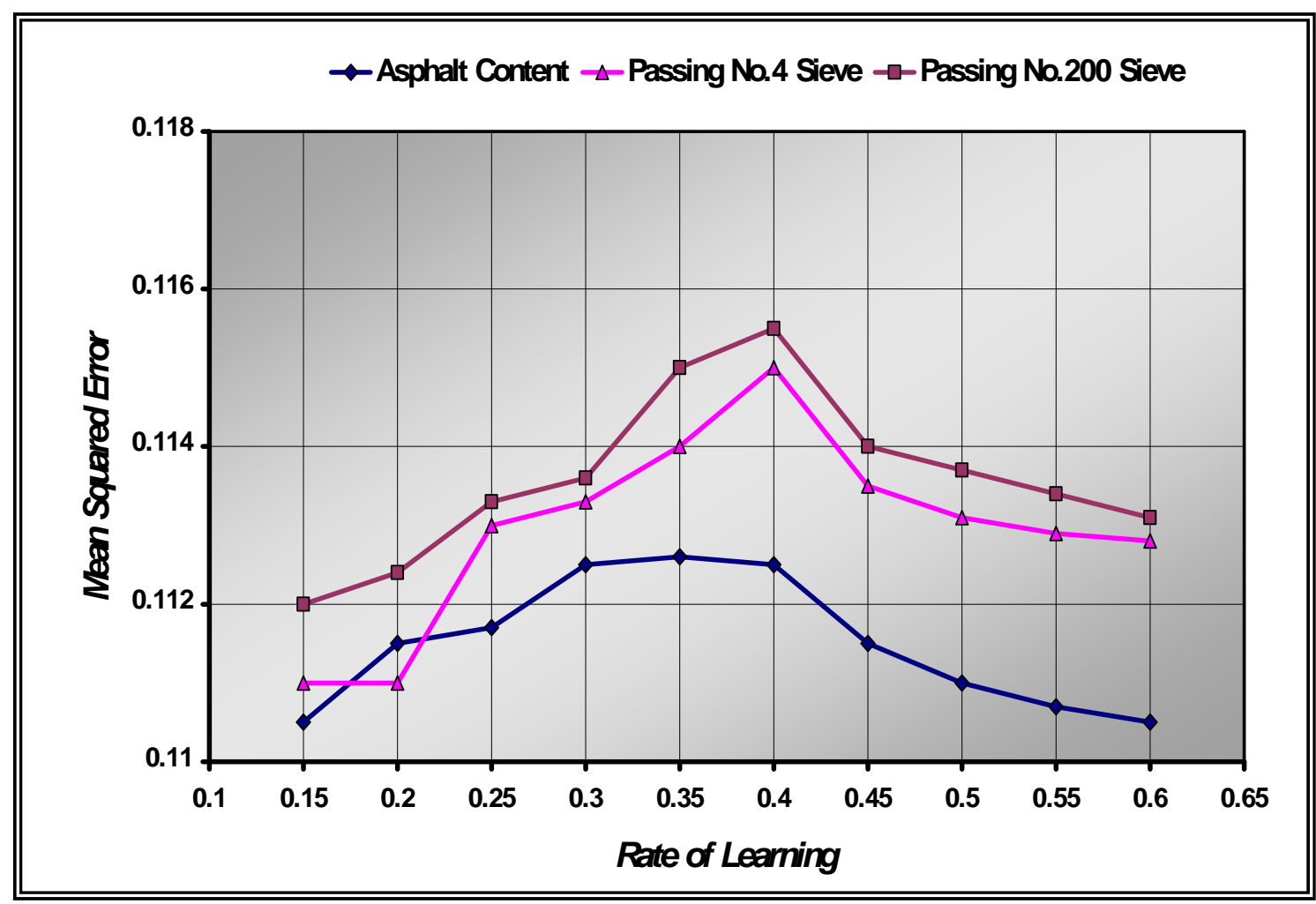

Figure 4. Mean Squared Error With Respect to Learning Rate for the Output Parameters of the study Network.

The selected network was trained for several epochs (the node threshold (bias) $\theta_{j}$ equal to one) with respect to the MSE, as presented in Figure 5, and the MSE against the number of epochs ( at the same learning rate ) for the predicted values of each output parameter are shown in the Figure 6. This figure shows that the training converged or the MSE decreased as the number of epochs were increased. With 1250 epochs and more, the MSE decreased slightly. 


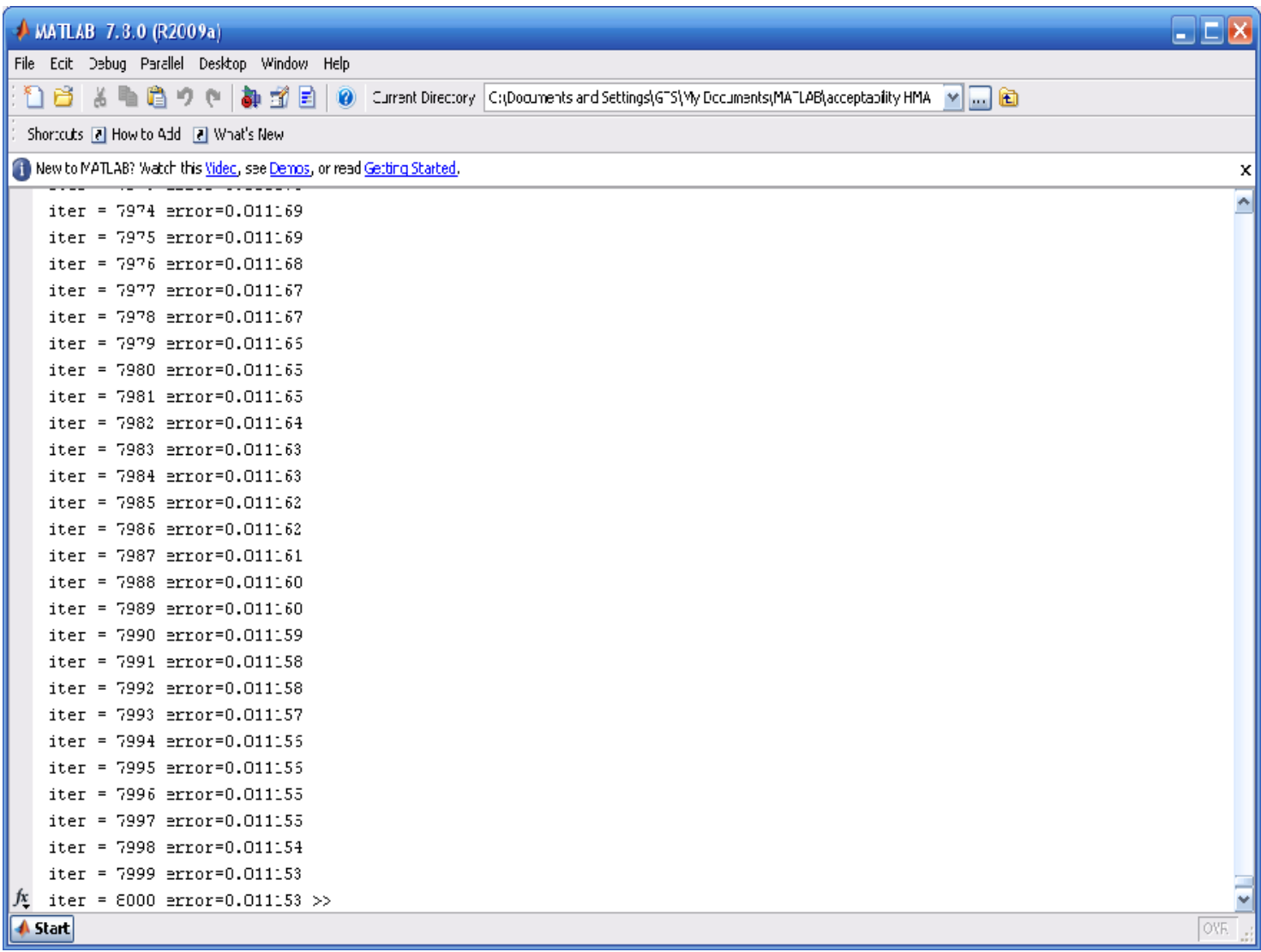

Figure 5. Mean Squared Error and Numbers of Epochs Relationships During the Study Network Run.

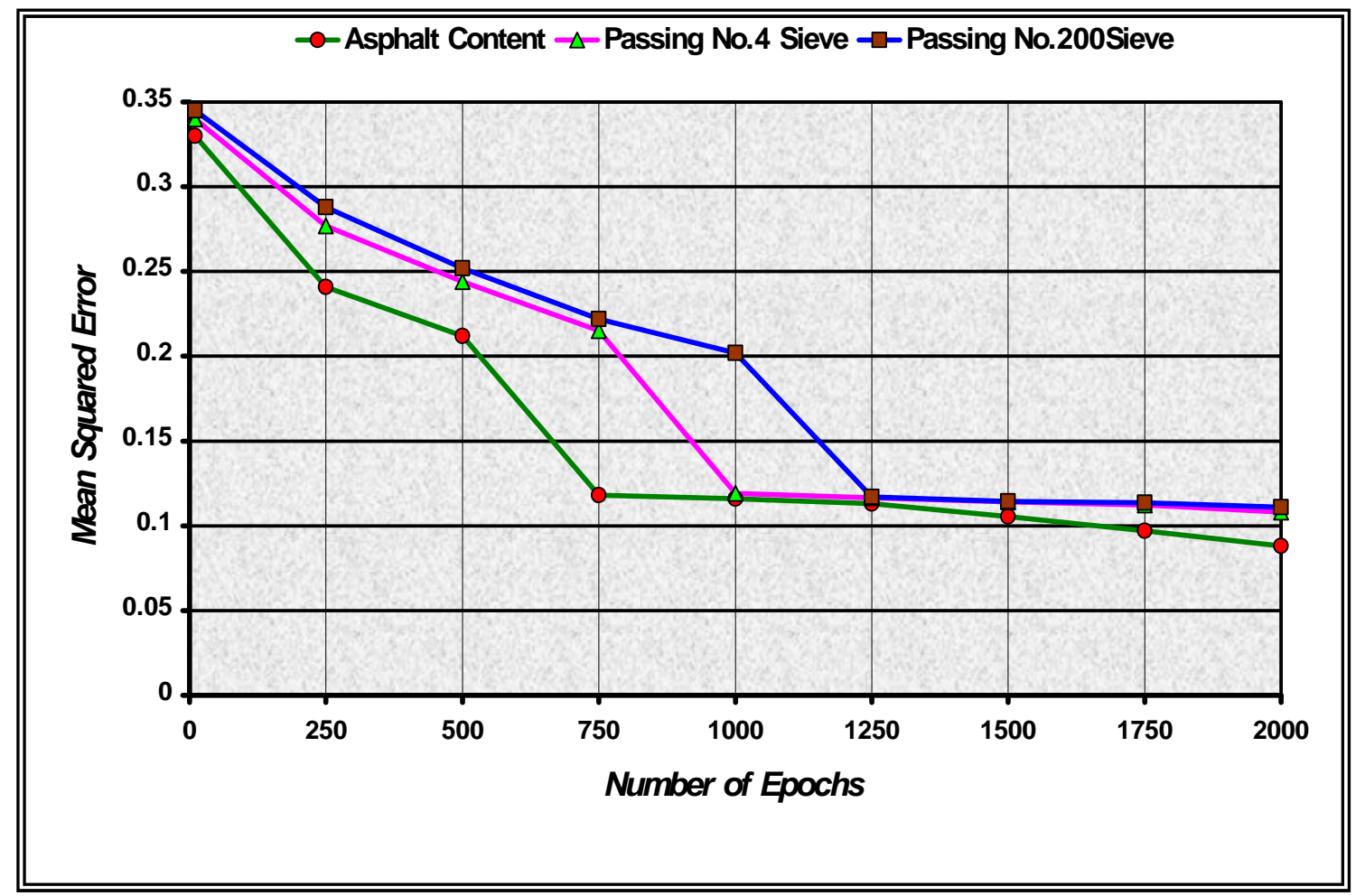

Figure 6. Mean Squared Error at Different Numbers of Epoch Output Parameters of the Study Network. 
Figures 7, 8 and 9 , describe the predicted values (outputs) versus the actual values (targets) of percent of asphalt, passing No.4 sieve and passing No.200 sieve respectively for both training and testing phases. The smaller spread of the result points would mean that predicted values of the output parameters are closer to the actual values and hence errors are small and vice-versa. These insignificant levels of MSE and high values of coefficient of determination $\left(R^{2}\right)$ are a good indication to degree of the applicability and acceptability of the network performance.

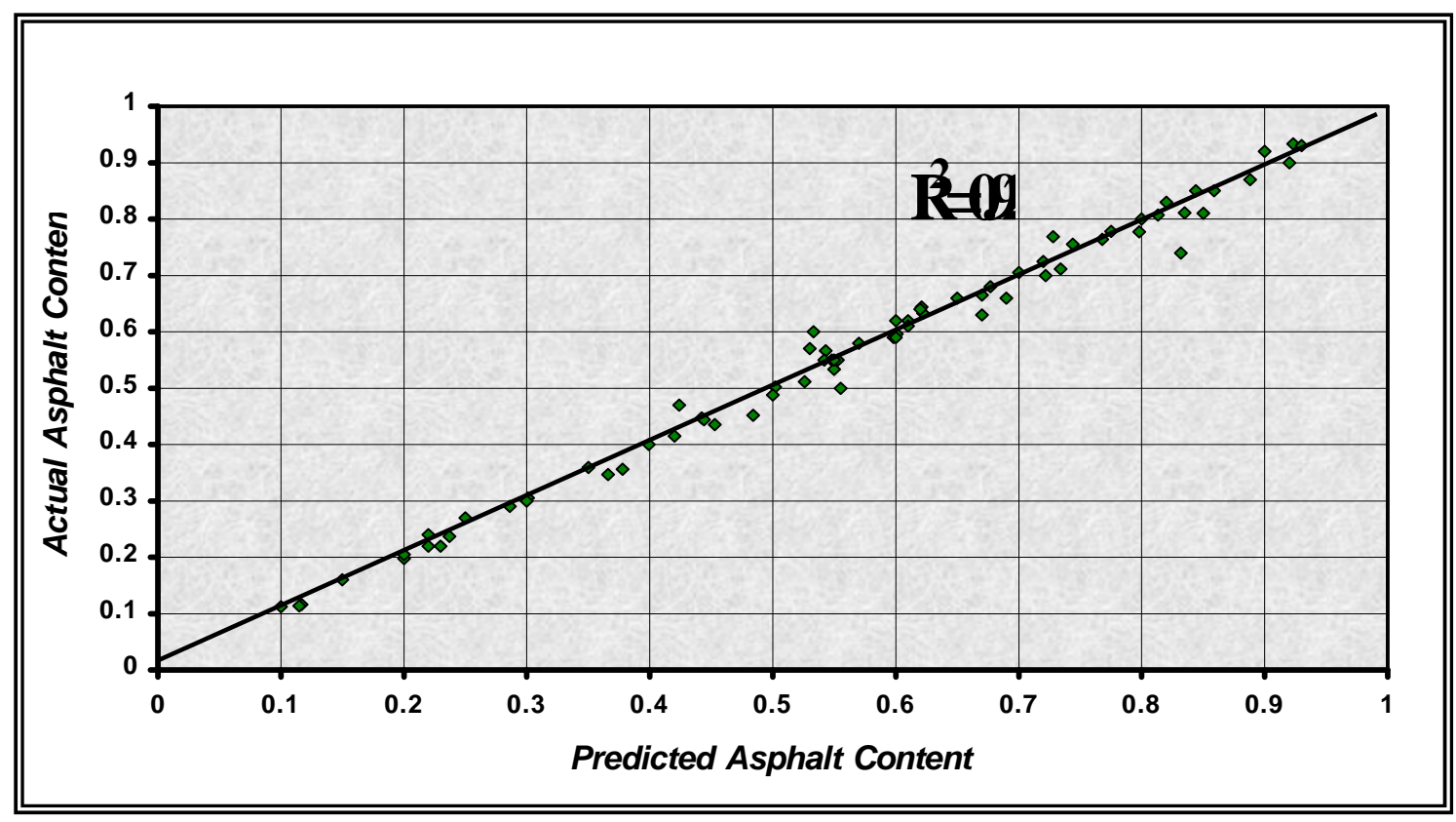

Figure 7. Actual Versus Study Network Predicted Asphalt Content Values.

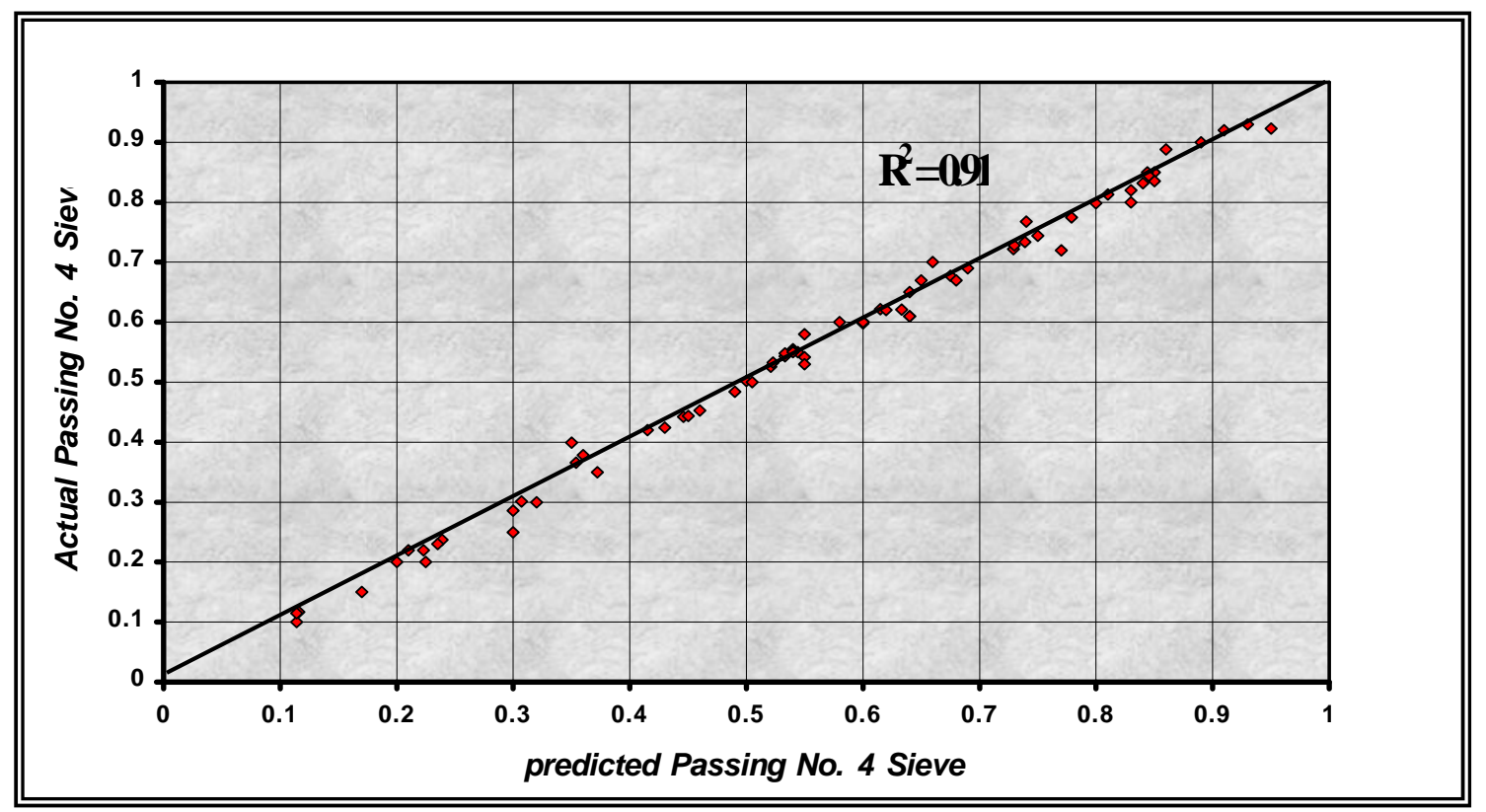

Figure 8. Actual Versus Study Network Predicted Passing No.4 Sieve Values. 


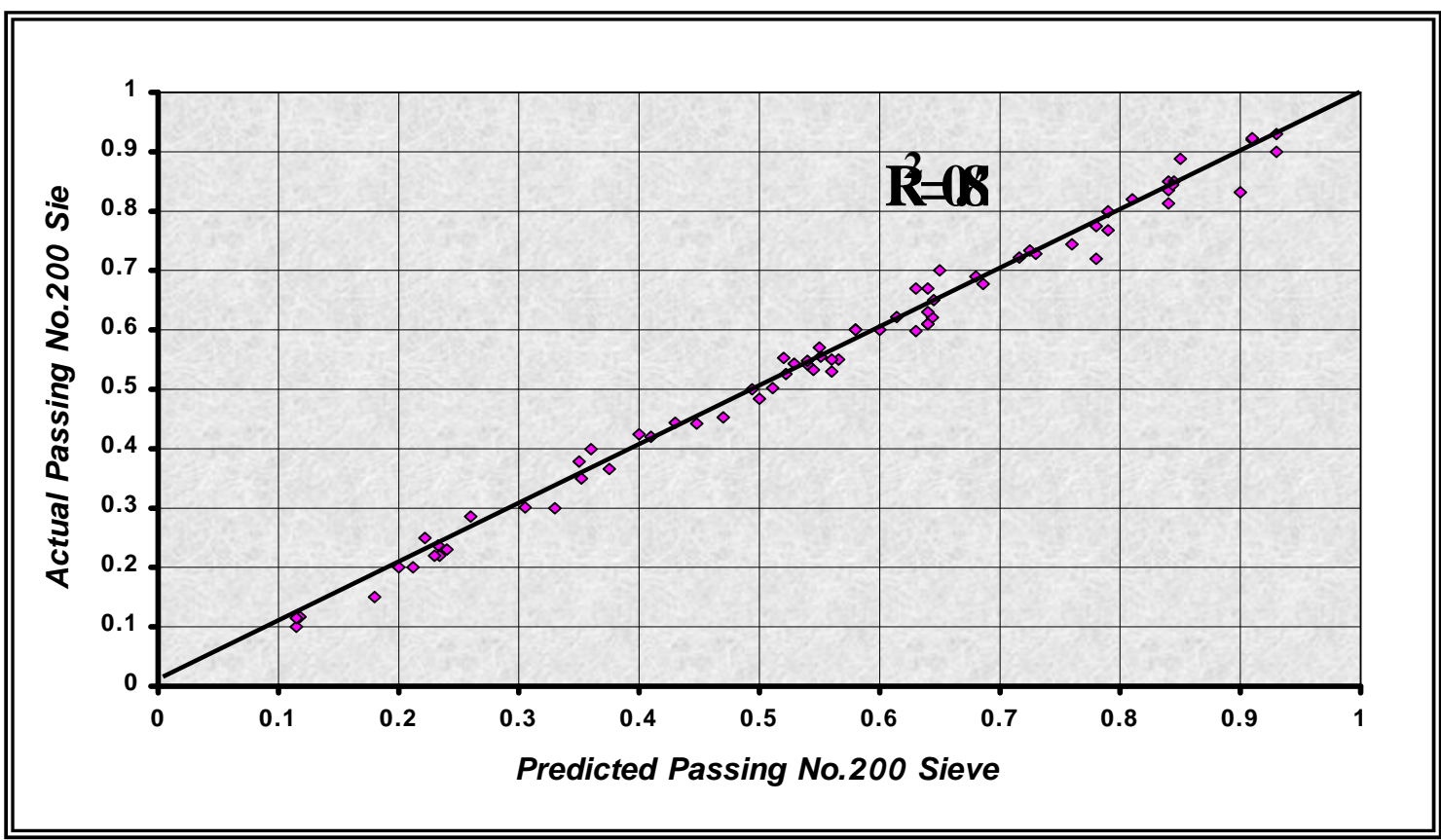

Figure9.Actual Versus Study Network Predicted Passing No.200 Sieve Values.

\section{Conclusions and recommendations}

This study examined the use of the backpropagation artificial neural network with MATLAB software for determining the acceptability of the HMA mixtures based on the prediction of the asphalt content and aggregate gradation, using the Marshall properties and specification requirements of these mixtures. According to the study results, the following conclusions and recommendations can be drawn:

1. As far as the present study is of concern, it developed the appropriate network structure which had the best performance with only 3 output parameters in the output layer. When the number of the output parameters are increased, the network structure and its operation became more complicated due to the increasing of the required number of nodes in the input layer, which mean the necessary need to increase the number of hidden layers and their nodes. As a result of this increasing, many problems may be caused related to network performance, speed of convergence or over-fitting failure.

2. This method can be used as an appropriated method for laboratories conducting quality control test and performing independent assurance, verification and acceptance testing.

3. Backpropagation was selected as the learning and training algorithm for ANN , because of its simplicity, strength in extracting useful information from examples, and its ability to store information in the form of connection weights. This model is easy to understand, and can be easily implemented as a software simulation.

4. Use of this method will be provide health and environmental benefits, cost saving, shorter test times and more acceptance results.

5. More work and development efforts still be required to evaluate this method under all conditions and limitations with various paving materials. Other efforts should also focus on the applicability and acceptability of the developed network to other pavement data. 
6. Future studies and efforts may be needed on investigating other types of ANN models and algorithms for analysis, design and testing problems in pavement structure, materials and construction.

\section{References}

1. Venayagamoorthy, V. and Allopi, D., ( 2007 ), " Use of Neural Networks in the Prediction of Bearing Capacity of Pavement Structures ", Proceedings of the $26^{\text {th }}$ Southern African Transport Conference, Pretoria, South Africa.

2. Tia, M., ( 2005 ), "Fundamental and Practice of Asphalt Mixture Design Procedures to Assure Adequate Performance ", Paper for presentation, $13^{\text {th }}$ Conference on Pavement Engineering, Taiwan.

3. Thomas, B., (2001), "Use of Artificial Neural Networks for Predicting Skid Resistance of Hot Mix Asphalt Concrete Pavements", Ph. D. Thesis, Texas Tech. University, Texas, USA.

4. Pekcan, O., Tutumluer, E. and Thompson, M. R., (2008), "Nondestructive Pavement Evaluation Using ILLI-PAVE Based Artificial Neural Network Models ", Civil Engineering Studies, Illinois Center for Transportation, Research Report FHWA-ICT-08-022.

5. Haykin, S.,(1999), "Neural Networks: A Comprehensive Foundation ", Second Edition, Prentice Hall Publication, Englewood Cliff, NJ., U.S.A.

6. Saghafi, B., Hassani, A., Noori, R. and Bustos, M., (2009), " Artificial Neural Networks and Regression Analysis for Predicting Faulting in Jointed Concrete Pavements Considering Base Condition", International Journal of Pavement Research and Technology, Vol. 2, No.1, pp. 20-25.

7. Rao, V. B. and Rao, H. V., (1993), "Neural Networks and Fuzzy Logic", Management Information Source, Inc., New York.

8. Fu, C.L., (1994), " Neural Networks in Computer Intelligence ", McGraw- Hill, Inc. New York.

9. Deshpande, N., ( 2008 ), " Artificial Intelligence ", $2^{\text {nd }}$ Revised, Technical Publication Pune, Pune, India.

10. Owusu-Ababia, S. ,(1998), " Effect of Neural Network Topology on Flexible Pavement Cracking Prediction ", Computer Aided Civil and Infrastructure Engineering, Vol. 13, Issue 5, pp. 295-303 .

11. Eldin, N. N. and Senouci, A. B., (1995)," A Pavement Condition Rating Model Using Backpropagation Neural Network ", Journal of Micro-Computer in Civil Engineering, Vol. 10, No. 6, pp. 433-441.

12. Choi, J. H., Adams, T. M., and Bahia, H. V., ( 2004 )," Pavement Roughness Modeling Using Back-Propagation Neural Networks ", Computer Aided Civil and Infrastructure Engineering, Vol. 19, Issue 4, pp. 295-303.

13. Venayagamoorthy, V., Allopi, D., and Venayagamoorthy, G. K. (2002),"Using Artificial Neural networks as a Design Tool for Flexible Pavement Design " , Proceeding of the IPET International Conference on Engineering Technolog y Research, pp. 58-61.

14. Venayagamoorthy, V., Allopi, D., and Venayagamoorthy,G. K. (2004),"Neural Network Based Classification For Pavement Structures ", Proceeding of the International Conference on Intelligent Sensing and Information Processing, India, pp. 295-298.

15. Pekcan, O., Tutumluer, E. and Thompson, M. R., ( 2006 ), " Nondestructive Flexible Pavement Evaluation Using ILLI-PAVE Based Artificial Neural Net- 
work Models ", ASCE, Atlanta, GA, USA, pp.227-232.

16. Attoh-Okine, N. O., ( 2001 ), " Grouping Pavement Condition Variables for Performance Modeling Using Self-Organizing Maps ", Journal of ComputerAided Civil and Infrastructure Engineering, Vol. 16, Issue 2, pp. 112-125.

17. Attoh-Okine, N. O. , ( 2002 ), " Combining Use of Rough Set and Artificial Neural Networks in Doweled-Pavement-Performance Modeling ", ASCE Journal of Transportation Engineering, Vol. 128, Issue3, pp.270-275.

18. Roberts, C.A., and Attoh-Okine,N.O., (1998), "A Comparative Analysis of Two Artificial Neural Networks Using Pavement Performance Prediction", Computer-Aided Civil and Infrastructure Engineering, Vol.13, Issue 5, pp. 339-348 .

19. Reddy, M. A., Murthy, M. S., and Pandey, B. B., (2002), " Back-calculation of Pavement Layer Moduli Using Genetic Algorithms ", Indian Roads Congress, Journal of Highway Research Board, No. 66, New Delhi, pp. 1-10.

20. Rakesh, N.,Jain,A. K., Reddy, M. A. and Reddy, K. S.,(2006),"Artificial Neural Networks: Genetic Algorithm Based Model For Backcalculation of Pavement Layer Moduli ",International Journal of Pavement Engineering, Vol. 7, No. 3, pp.221-230.

21. Alsugair , A. M. and Al-Qudrah, A. A. , ( 1998 )," Artificial Neural Network Approach For Pavement Maintenance ", Journal of Computing in Civil Engineering, ASCE, Vol. 12, No.2, pp. 249-255.

22. Fwa,T. F. and Chan, W.T., (1993), " Priority Rating of Highway Maintenance Needs by Neural Networks", ASCE Journal of Transportation Engineering, Vol. 119, pp.419-422.

23. Ceylan, H., et. al.,(2004), "Neural Network-Based Structural Models for Rapid Analysis of Flexible Pavement with Unbounded Aggregate Layers",Proceeding of $6^{\text {th }}$ International Symposium on Pavements Unbounded, Nottingham, England, pp. 139-147.

24.Goktep,A.B.,Agar, E., Lav, A.H.,(2004),"Comparison of Multilayer Preceptron and Adaptive Neuro-Fazzy System on Back-calculating the Mechanical Properties of Flexible Pavement", ARI, the Bulletin of the Istanbul Technology University, Vol. 54, No. 3, Istanbul, pp.65-77.

25. Saltan, M., and Terzi, S. , ( 2004 ), " Backcalculation Of Pavement Layer Parameters Using Artificial Neural Networks", Indian Journal of Engineering and Materials Sciences, Vol. 11, No. 1, pp. 38-42.

26. Ozsahin, T. S. and Oruc, S., ( 2008 ), " Neural Network Model for Resilient Modulus of Emulsified Asphalt Mixtures ", Construction and Building Materials, Vol. 22, Issue 7, July 2008, pp. 1436-1445.

27. Kaseko,M. S., Ritchie,S.G.,and Lo,Z. P.,(1993),"Evaluation of Two Automated Thresholding Techniques for Pavement Images",Infra-Structure Planning and Management Conference Proceedings, Denver, Colorado, pp. 277-281.

28. Guralnick, S. A., Suen, E. S. and Gu, J., ( 1993 ), "Neural Network System for Automated Highway Pavement Inspection ", Infrastructure Planning and Management Conference Proceedings, Denver, Colorado, pp.272-276.

29. Manik, A., Gopalakrishnan,K., Singh, A. and Yan, S.,(2008),"Neural Networks Surrogate Models for Simulating Payment Risk in Pavement Construction ", Journal of Civil Engineering and Management, Vol. 14, No.4, pp. 235-240.

30. Goktepe,A.B., Agar,E. and Lav,A H.,(2006),"Advances In Backcalculating the Mechanical Properties of Flexible Pavements", Advances in Engineering Software, Vol. 37, Issue 7, pp. 421-431.

31. Sarkar D., ( 1995 ), " Methods to Speed up Error Backpropagation Learning 
Algorithm ", ACM Computing Surveys, Vol. 27, No. 4, pp. 519-541.

32. Nawi,N. M.,Ransing, R. S. and Ransing,M. R.,(2008),"An Improved Conjugate Gradient Based Learning Algorithm for Back-Propagation Neural Networks", International Journal of Computational Intelligence, Vol.4, No.1 , pp. 46-55.

33. Gonzalez, R. C., Woods, R. E., and Eddins, S. L., ( 2008 ) , " Digital Image Processing Using MATLAB", Pearson Prentice Hall, New Jersey.

34. Republic of Iraq, Ministry of Housing and Construction, (2003)," General Specification for Roads and Bridges ", Revised Edition 2003, Department of Planning and Studies, Iraq.

35. American Society for Testing and Materials, ASTM, (2004), "Annual Book of ASTM Standards", PA, U.S.A. 